\title{
Contact investigation of a case of human novel coronavirus infection treated in a German hospital, October-November 2012
}

U Buchholz (buchholzu@rki.de)1,2, M A Müller ${ }^{3,2}$, A Nitsche $^{1,2}$, A Sanewski 4,2, N Wevering ${ }^{5}$, T Bauer-Balci ${ }^{6}$, F Bonin ${ }^{5}$, C Drosten

(drosten@virology-bonn.de) ${ }^{3}$, B Schweiger ${ }^{1}$, T Wolff ${ }^{1}$, D Muth ${ }^{3}$, B Meyer ${ }^{3}$, S Buda $^{1}$, Krause $^{1}$, L Schaade ${ }^{1}$, W Haas $^{1}$

1. Robert Koch Institute, Berlin, Germany

2. These authors contributed equally to this work

3. University Bonn, Department of Virology, Bonn, Germany

4. County health department of Essen, Essen, Germany

5. Ruhrland hospital, Essen, Germany

6. County health department Oberbergischer Kreis, Gummersbach, Germany

Citation style for this article:

Buchholz U, Müller MA, Nitsche A, Sanewski A, Wevering N, Bauer-Balci T, Bonin F, Drosten C, Schweiger B, Wolff T, Muth D, Meyer B, Buda S, Krause G,

Schaade L, Haas W. Contact investigation of a case of human novel coronavirus infection treated in a German hospital, October-November 2012. Euro Surveill.

2013;18(8):pii=20406. Available online: http://www.eurosurveillance.org/ViewArticle.aspx?Articleld=20406

Article submitted on 24 January 2013 / published on 21 February 2013

On 24 October 2012, a patient with acute respiratory distress syndrome of unknown origin and symptom onset on 5 October was transferred from Qatar to a specialist lung clinic in Germany. Late diagnosis on 20 November of an infection with the novel Coronavirus ( $\mathrm{NCoV}$ ) resulted in potential exposure of a considerable number of healthcare workers. Using a questionnaire we asked 123 identified contacts (120 hospital and three out-of-hospital contacts) about exposure to the patient. Eighty-five contacts provided blood for a serological test using a two-stage approach with an initial immunofluorescence assay as screening test, followed by recombinant immunofluorescence assays and a NCoV-specific serum neutralisation test. Of 123 identified contacts nine had performed aerosol-generating procedures within the third or fourth week of illness, using personal protective equipment rarely or never, and two of these developed acute respiratory illness. Serology was negative for all nine. Further 76 hospital contacts also tested negative, including two sera initially reactive in the screening test. The contact investigation ruled out transmission to contacts after illness day 20. Our two-stage approach for serological testing may be used as a template for similar situations.

\section{Introduction}

A novel human coronavirus ( $\mathrm{NCoV}$ ) has recently emerged in the Arabian Peninsula. The first two reported cases infected by the novel agent, then provisionally termed hCoV-EMC, occurred in June and September 2012, respectively [1-3]. As of 18 February 2013, a total of 12 cases have been confirmed by WHO [4], including five deaths. Among five cases reported from the Kingdom of Saudi Arabia, three were part of a family cluster. Two further cases were linked to probable exposure in Qatar, and two cases were confirmed retrospectively, by diagnostic of respiratory specimens, from Jordan with disease onset in April 2012. The latter were part of a cluster of 11 patients with acute respiratory symptoms linked to a hospital [5]. The most recent three cases identified constitute another cluster that occurred in the United Kingdom (UK) in January to February 2013 [4]. The index case in this cluster is a UK citizen with travel history to SaudiArabia and Pakistan before symptom onset [5]. Two of his family members who had not travelled outside the UK and became ill were most likely infected through person-to-person transmission. While one of them had an underlying disease and died, the other presented with milder, influenza-like illness symptoms only.

Because of the long period, 10 months, over which the cases occurred, the source and transmission patterns of the virus remain elusive. Hypotheses include a predominance of zoonotic acquisitions with little potential for human-to-human transmission [5], widespread and unnoticed occurrence of clinically mild infections, and finally the possibility of an early-stage epidemic caused by a highly pathogenic novel human virus.

Because of the potential of human-to-human transmission in the hospital outbreak in Jordan and the family clusters, as well as the observed severity of disease, current recommendations regarding protective measures rely on experiences with severe acute respiratory syndrome (SARS) in 2003 [6]. The first of the two Qatari patients was treated in the UK where, under strict isolation measures, no secondary cases occurred. Investigations by polymerase chain reaction (PCR) of 10 healthcare workers (HCW) who had cared for the patient and subsequently developed mild respiratory disease yielded no evidence of infection [7]. However, to date, published investigations of individuals with proven exposure to $\mathrm{NCoV}$ have not presented 
Timeline of disease of novel coronavirus case and possible exposure of healthcare workers, Germany October-November 2012

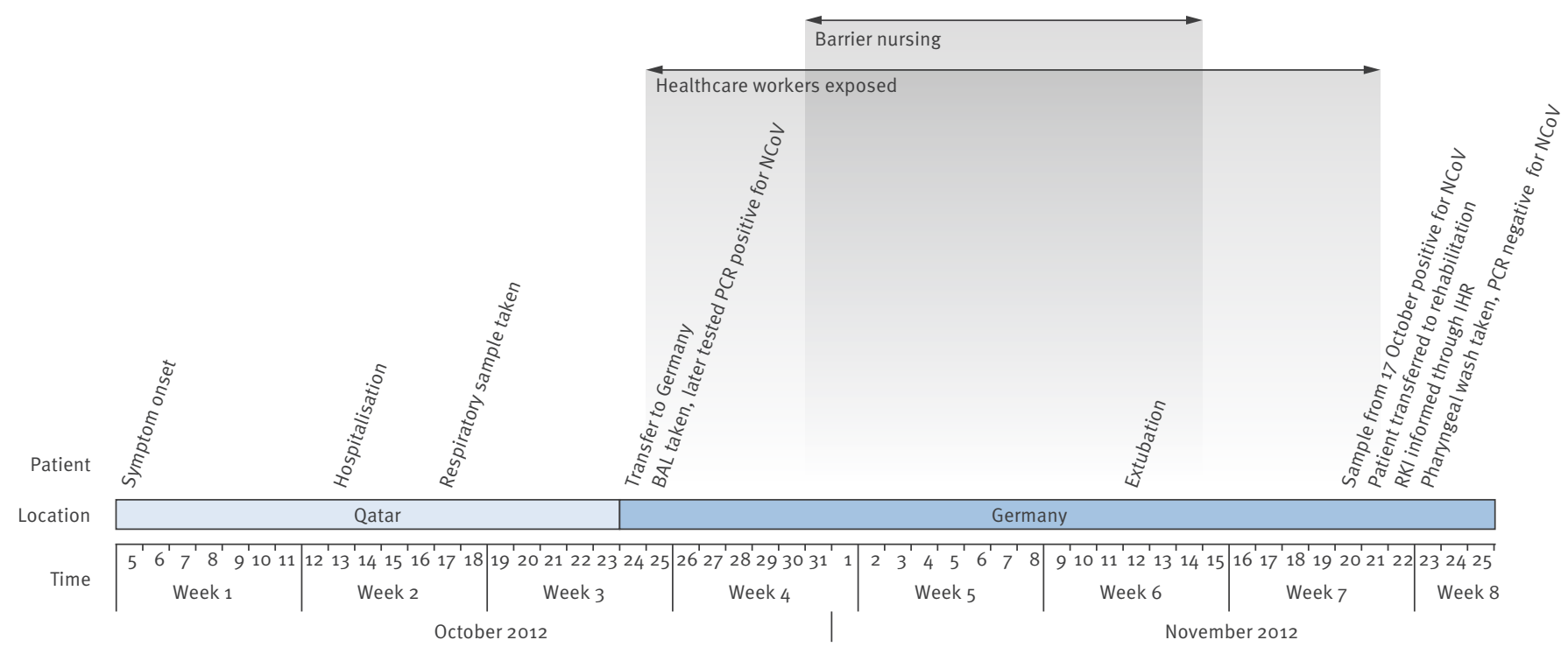

BAL: bronchoalveolar lavage; IHR: International Health Regulations; NCoV: novel coronavirus; PCR: polymerase chain reaction; RKI: Robert Koch Institute.

a strategy how to identify retrospectively infections in a large group of (contact) persons through serological testing.

On 22 November 2012, the Robert Koch Institute in Berlin, Germany, was informed according to the International Health Regulations [8] about a case of NCoV infection in a Quatari patient in his forties, treated in Germany (Figure). After an acute onset of symptoms on 5 October, he had been admitted to a hospital in Doha, Qatar, on 13 October, where he developed respiratory failure requiring ventilation, and was reported to have had temporary renal impairment. On 24 October, he was transferred to a specialist lung hospital in Essen, Germany. A respiratory sample had been taken in Qatar on 17 October. After some delay due to difficulties with the shipment of specimens, the sample tested positive for NCoV in a laboratory in the UK. The result was consequently communicated by the UK Health Protection Agency to the World Health Organization (WHO) on 21 November 2012. Until that date the hospital in Essen had not considered NCoV in the differential diagnoses for the patient. Only routine personal protection of $\mathrm{HCW}$ and no specific measures of respiratory protection had been followed during the whole course of treatment in the intensive care unit (ICU). After weeks of mechanical ventilation in ICU, the patient was discharged on 21 November. A lag time of four weeks between patient transfer and laboratory confirmation of the NCoV infection resulted in potential exposure of a considerable number of HCW in Germany. Here we report on an interview with the patient asking for potential sources of infection, the investigation of individuals exposed to the patient, virological investigation of respiratory samples from the patient as well as an approach used to test retrospectively a large number of contacts.

\section{Methods}

Patient interview and samples for

laboratory investigation

After the patient had recovered he was interviewed in person. The interview was conducted in Arabic with the help of an interpreter. It was targeted at potential modes of acquisition of the infection. The questionnaire contained questions about the early course of disease, social status, living conditions, profession, hobbies and regular activities, exposure to animals, eating habits, and contacts with individuals with respiratory illness in the 10 days before his illness onset.

We searched for stored respiratory and blood samples at the hospital laboratory that were still available to be tested for $\mathrm{NCoV}$ and identified a specimen that originated from a bronchioalveolar lavage (BAL) done on 25 October, illness day 20 (i.e. late third illness week), as well as a serum sample from the same day. In addition, on 23 November (eighth illness week), we took a pharyngeal wash and a serum sample from the patient after he was discharged and had started his rehabilitation program on 21 November. Both respiratory samples were tested by real-time reverse-transcription (RT)$P C R$. The first sample was also subjected to virus isolation in LLC-MK2 cells. 


\section{Contact investigation}

Contact persons were identified based on electronic procedures registration, supplemented by a targeted request to $\mathrm{HCW}$ to report contact with the patient while in ICU (and during the transport to the hospital). The electronic registration of procedures requires that any person performing a task in the patient's room must sign in, sign out and document which procedure was conducted. Using a standardised questionnaire, information was collected about the time of the first contact, types of contact, closest distance to the patient, frequency of using a surgical face mask when in contact with the patient, and occurrence of acute respiratory illness (ARI) up to ten days after the last contact with the patient. No information was collected on the duration or frequency of contact.

Consenting individuals gave blood for serological testing on one of three dates ( 3,7 or 14 December). The median interval from first patient contact to venipuncture was 39 days (range: 13-50 days). Contacts were considered at high risk if they had their first contact with the patient at the beginning of his stay in ICU, i.e. at the end of the patient's third or fourth week of illness, if they had conducted an aerosol-generating procedure, such as suctioning the intubated patient or performing a BAL, and if they had rarely or never used surgical face masks while caring for the patient.

\section{Laboratory methods}

Nucleic acid detection was performed by RT-PCR as described previously $[9,10]$ after viral RNA was extracted from $300 \mu \mathrm{l}$ of bronchioalveolar lavage using the MagAttract Viral RNA Kit M48 (Qiagen GmbH, Hilden, Germany).

Serological testing was performed in a two-stage approach. As a first step, screening for antibodies reactive to $\mathrm{NCoV}$ was done by indirect immunofluorescence assay (IFA) as described previously [10]. Preliminary evaluation of IFA on 50 sera from blood donors yielded no reactivity. For resolution of reactive results, IFA was done on Vero B4 cells expressing recombinant spike $(\mathrm{S})$ and nucleocapsid $(\mathrm{N})$ proteins of $\mathrm{NCoV}, \mathrm{SARS}-\mathrm{CoV}, \mathrm{hCoV}-\mathrm{OC} 43$, and hCoV-NL63. Details of procedures for recombinant IFA are outlined in Corman et al. [10]. For serum neutralisation tests (SNT), Vero B4 cells were grown to subconfluence in 24 well plates. Preincubation involved 25 plaque-forming units of $\mathrm{NCoV}$ in $100 \mu \mathrm{l}$ of medium, mixed 1:1 with patient sera prediluted in medium as indicated. The starting dilution was $1: 8$. After $1 \mathrm{~h}$ incubation at $37{ }^{\circ} \mathrm{C}$, each well was infected for $1 \mathrm{~h}$ at $37^{\circ} \mathrm{C}$ using the total 200 $\mu \mathrm{l}$ preincubation reaction. Supernatants were removed and overlaid with Avicell resin exactly as described by Herzog et al. [11]. Assays were terminated and stained after three days.

\section{Statistical tests}

Comparison of frequency distributions were done using Fisher's exact test.
Ethical clearance and data protection

The contact investigation was carried out based on legal requirements of the Protection against Infection Act of Germany [12] and the International Health Regulations [8], and was led by the local health authorities. After information about the investigation and its aims, contacts signed a consent form if they agreed with the analysis of blood samples. All questionnaires and samples were fully anonymised before analysis.

\section{Results}

\section{Patient interview}

The patient reported to live in Doha, Qatar. He used to be a heavy smoker ( 2 to 3 packs of cigarettes per day), but denied smoking waterpipe or chewing qat. Disease onset was rapid, with initial symptoms including fever $\left(40^{\circ} \mathrm{C}\right)$, cough, runny nose, and shortness of breath. Subjective weakness was pronounced. After the first two days of illness he improved a little but deteriorated again, and was finally admitted to hospital on day eight of illness because of increasing dyspnoea. He reported no subjective symptoms of renal impairment such as foamy urine, reduced urine output, or back pain. He had not travelled and had no known contact with any other reported cases of NCoV infection. The patient owned a camel and goat farm and reported a large number of casual contacts (approx. 50 persons per day) on a regular basis. He remembered that before his disease onset some goats were ill and had fever. He did not have direct contact with the goats or any other animals especially falcons or bats, but said he had eaten goat meat. He also reported to have had contact with one of his animal caretakers who was ill with severe cough and was hospitalised. Other than the animal caretaker, he did not remember persons with severe respiratory illnesses in his wider or closer social environment.

\section{Patient samples}

Virus detection in the initial sample from illness day 20 and preliminary serological investigations have been described by Corman et al. [10]. Isolation of virus in cell culture failed. Serological testing yielded an IgM titre against NCoV of 1:1,000 and an IgG titre of 1:10,000 at day 20 (week three) of illness. At week eight of illness the IgG titre was still at 1:10,000 while the IgM titre had already decreased to 1:100. SNT titres against NCoV were 1:640 at week three and 1:640 at week eight of illness. The pharyngeal wash sample taken on 23 November 2012 (week eight of illness) tested negative by real-time RT-PCR.

\section{Contact investigation}

We identified 120 hospital and three out-of-hospital contacts, including the interpreter of the patient. Protective measures were largely limited to HCW wearing gloves and gowns when providing intimate care and use of surgical face masks during suctioning. From 31 October until 4 November (illness weeks five and six), the patient was isolated using barrier nursing due to 
a concurrent Pseudomonas aeruginosa infection. This included use of surgical masks only. Among the 120 hospital contacts the largest group were nurses $(n=59$; $49 \%)$, followed by physicians ( $n=26 ; 22 \%)$ and laboratory technicians $(n=15 ; 13 \%)$ (Table 1$)$. Median time from first contact to venipuncture was 39 days (range: 13-50 days).

Eighty-five (69\%) of all respondents reported contact at a distance of less than or equal to $2 \mathrm{~m}, 14$ (11\%) of more than $2 \mathrm{~m}$, and $24(20 \%)$ of unknown distance to the patient. Frequency of ARI by week of first contact differed significantly among the groups (Table 1). However, there was no trend in the ARI proportion over time: eight of 33 contacts with first exposure during illness weeks three or four experienced ARI within 10 days of last contact; five of nine contacts with first exposure during the patient's fifth week of illness; and none of 14 with first contact during week six of illness developed ARI.

Among 81 contacts reporting exposure within $2 \mathrm{~m}, 21$ had ARI compared to none of 14 with contact of more than $2 \mathrm{~m}$ ( $p$ value; 0.04) (Table 1). Among those with first exposure in week three or four of illness of the patient, the proportion of contacts with ARI was not significantly different between those considered to be at high risk and the remaining contacts ( $p$ value, 0.87) (Table 1). Thirteen HCW had contact to the patient in weeks three or four of illness, had contact within $2 \mathrm{~m}$ to the patient and had worn surgical face masks rarely or never. Among these, nine were high-risk contacts, including one nurse who assisted in a bronchoscopy on 25 October. All nine provided a blood sample. The median time after last contact with the patient for these nine HCW was 32 days (range: 13-46 days). No sample was reactive by IFA.

Of the remaining 76 blood samples, one serum showed reactivity for IgM even at dilutions up to 1:100. This titre could be resolved as a cross-reacting recent infection with hCoV-NL63 by IFA using recombinant $\mathrm{S}$ and $\mathrm{N}$ proteins from major hCoVs (Table 2), as well as absence of NCoV-specific neutralising antibodies. Another serum showed indeterminate IgG-reactivity in a 1:10 dilution. Specific anti-NCoV antibodies were ruled out by recombinant IFA, indicating earlier infection with hCoV-OC 43 and hCoV-NL63, as well as absence of any significant titre in SNT (Table 2).

\section{Discussion}

Here we describe a case and contact investigation of a laboratory-confirmed patient with NCoV infection for whom the suspicion of this possible aetiology had not been discussed with the treating hospital upon admission of the patient. The patient still tested PCR-positive late in his third week of illness. Despite this we concluded from the laboratory findings that his infectiousness was then absent or very low. While at that time no consistent dedicated personal protective measures had been applied by HCW caring for the patient, our public health investigation did not show infection in any of the 85 serologically tested contact persons, mainly HCW. The conducted serological two-stage approach was an effective method of screening a large number of contact persons for infection.

For initial risk assessment, after the information in November about the cause of the patient's disease, it was important to know if he had been potentially infectious at the time of arrival at the hospital in Germany in October. One stored respiratory sample taken at the time of admission, yielded clear, albeit very low quantities of NCoV RNA in the range of 66.5 to 100 copies per $\mathrm{mL}$ [10]. Attempts to isolate virus from this sample were unsuccessful. Even though the sample had been stored for prolonged time under less-than-optimal conditions, these combined RT-PCR and cell culture data suggested absent or very low infectiousness at the time of admission. Negative RT-PCR four weeks later, just after discharge from hospital, suggested the patient had cleared the virus, and no further respiratory precautions were necessary upon admission to the rehabilitation centre.

Nevertheless, anxiety and lack of any other epidemiological data made it necessary to gauge rapidly the significance of some cases of ARI experienced in HCW who had been in contact with the patient. Our data yielded no direct correlation of ARI rates with time of exposure. In particular, those contacts considered at highest risk had no more ARI than other contacts who also had their first contact with the patient during the third or fourth illness week.

In the context of a retrospective contact investigation, our two-staged serological approach proved effective in ruling out any NCoV infections among contacts including those who developed acute respiratory disease. Preliminary screening using a generic serological test provides a reliable result for negative samples. Hereafter only positive or indeterminate results need to be further scrutinised using the described methods.

During two interviews that the patient kindly agreed to, we explored a wide spectrum of factors that he might have been exposed to. Even though NCoV is genetically similar to bat coronaviruses $[1,13,14]$, other animals may serve as (intermediate) host as well. While our patient denied contact to bats, he remembered ill goats among the animals on his farm. Albarrak et al. reported that the first Saudi case was exposed to farm animals, but the first Qatari patient and the second Saudi patient were not [15]. Although our patient reported no direct contact with his animals, one animal caretaker working for him was ill with cough and might have been an intermediate link in the chain of infection.

Coronaviruses do infect ruminants such as goats [16] and thus goats could be considered as a possible source of origin for the novel virus, particularly in 
TABLE 1

Profession, type of contact, occurrence of acute respiratory illness and serological results in contacts of case of novel coronavirus infection, Germany October-November 2012

\begin{tabular}{|c|c|c|c|c|}
\hline Variable & $\mathrm{N}$ & $\begin{array}{c}\mathrm{N} \text { (with } \\
\text { information) }\end{array}$ & $\%$ & Pvalue \\
\hline Contacts & 123 & 123 & 100 & - \\
\hline Hospital staff & 120 & 123 & 98 & - \\
\hline Out-of-hospital persons & 3 & 123 & 2 & - \\
\hline Response to questionnaire & 110 & 123 & 89 & - \\
\hline Acute respiratory infection & 24 & 104 & 23 & - \\
\hline Provided blood sample & 85 & 123 & 69 & - \\
\hline Interval between first contact and venipuncture $(n=48)$ & \multicolumn{3}{|c|}{ median: 39 days (range: $13-50$ ) } & - \\
\hline \multicolumn{5}{|l|}{ Serology for antibodies against NCoV } \\
\hline Positive & 0 & 85 & 0 & - \\
\hline Negative & 85 & 85 & 100 & - \\
\hline \multicolumn{5}{|l|}{ Professional group among hospital staff } \\
\hline Nursing staff & 59 & 120 & 49 & - \\
\hline Physicians & 26 & 120 & 22 & - \\
\hline Laboratory technicians & 15 & 120 & 13 & - \\
\hline Physician and team assistants & 13 & 120 & 11 & - \\
\hline Physiotherapists & 4 & 120 & 3 & - \\
\hline House maintenance & 4 & 120 & 3 & - \\
\hline Cleaning staff & 2 & 120 & 2 & - \\
\hline \multicolumn{5}{|l|}{ Contact distance to patients } \\
\hline$\leq 2$ metres & 85 & 123 & 69 & - \\
\hline$>2$ metres & 14 & 123 & 11 & - \\
\hline Unknown & 24 & 123 & 20 & - \\
\hline \multicolumn{5}{|l|}{ First contact in the 3 rd or $4^{\text {th }}$ week of patient's illness } \\
\hline Yes & 36 & 123 & 29 & - \\
\hline Later or unknown & 87 & 123 & 71 & - \\
\hline \multicolumn{5}{|l|}{ ARI by contact distance to patients } \\
\hline$\leq 2$ metres & 21 & 81 & 26 & \multirow{2}{*}{0.04} \\
\hline$>2$ metres & 0 & 14 & 0 & \\
\hline \multicolumn{5}{|l|}{ ARI by week of first contact } \\
\hline 3rd/4th illness week & 8 & 33 & 24 & \multirow{3}{*}{$<0.01$} \\
\hline 5th illness week & 5 & 9 & 56 & \\
\hline 6th illness week or later & 0 & 17 & 0 & \\
\hline \multicolumn{5}{|l|}{ ARI in those exposed in 3rd or 4th week, by risk level } \\
\hline $\begin{array}{l}\text { High risk, i.e. performing aerosol-generating procedures, } \\
\text { face mask rarely/not worn }\end{array}$ & 2 & 9 & 22 & \multirow[t]{2}{*}{0.87} \\
\hline All others & 6 & 24 & 25 & \\
\hline \multicolumn{5}{|l|}{ High-risk contacts ${ }^{\mathrm{b}}$} \\
\hline who provided blood & 9 & 9 & 100 & - \\
\hline Interval between last contact and venipuncture $(n=9)$ & \multicolumn{3}{|c|}{ median: 27 days (range: $12-46$ ) } & - \\
\hline
\end{tabular}

ARI: acute respiratory illness; NCoV: novel coronavirus.

a Based on Fisher's exact test.

b A high-risk contact is a contact who had contact in the (3rd or) 4th week of the patient's illness, performed aerosol-generating procedures and wore face mask rarely or not at all. 
Cross-reactivity test on contact persons and of case of novel coronavirus infection (at week 3 and week 8 of illness) with recombinant spike and nucleocapsid indirect fluorescence antibody test ${ }^{\mathrm{a}}$, Germany October-November 2012

\begin{tabular}{|c|c|c|c|c|c|c|}
\hline Virus & Antibody type & Contact 1 & Contact 2 & $\begin{array}{l}\text { Patient } \\
\text { (week 3) }\end{array}$ & $\begin{array}{l}\text { Patient } \\
\text { (week 8) }\end{array}$ & Negative control $^{\mathrm{b}}$ \\
\hline \multicolumn{7}{|l|}{$\mathrm{NCoV}$} \\
\hline \multirow{2}{*}{ Spike } & $\lg M^{c}$ & $+/-$ & $+/-$ & $21: 320^{d}$ & $>1: 320$ & - \\
\hline & $\lg G$ & - & - & $21: 320$ & $>1: 320$ & - \\
\hline \multirow{2}{*}{ Nucleocapsid } & $\lg M$ & - & $1: 20$ & ND & - & - \\
\hline & $\lg G$ & - & $+/-$ & ND & + & - \\
\hline \multicolumn{7}{|l|}{ SARS-CoV } \\
\hline \multirow{2}{*}{ Spike } & $\lg M$ & $+/-$ & - & ND & + & - \\
\hline & $\lg G$ & - & - & ND & - & - \\
\hline \multirow{2}{*}{ Nucleocapsid } & $\lg M$ & - & - & ND & - & - \\
\hline & $\lg G$ & - & - & ND & - & ++ \\
\hline \multicolumn{7}{|l|}{ hCoV-OC43 } \\
\hline \multirow{2}{*}{ Spike } & $\lg M$ & $+/-1+$ & - & + & + & - \\
\hline & $\lg G$ & ++ & $>1: 80$ & ++ & ++ & +++ \\
\hline \multirow{2}{*}{ Nucleocapsid } & $\lg M$ & - & - & $+/-$ & $+/-$ & - \\
\hline & $\lg G$ & $+/-1+$ & $+/-$ & $+/-1+$ & $+/-1+$ & + \\
\hline \multicolumn{7}{|l|}{ hCoV-NL63 } \\
\hline \multirow{2}{*}{ Spike } & $\operatorname{Ig} M$ & $1: 80$ & $+/-$ & $1: 80$ & $>1: 320$ & - \\
\hline & $\lg G$ & $>1: 320$ & $1: 20$ & $21: 320$ & $>1: 320$ & - \\
\hline \multirow{2}{*}{ Nucleocapsid } & $\lg M$ &.- & - & ND & - & - \\
\hline & $\lg G$ & ++ & $+/-$ & ND & $+1-1+$ & - \\
\hline
\end{tabular}

CoV: Corona virus; hCov: human coronavirus; NCoV: novel coronavirus; ND: not done; SARS: severe acute respiratory syndrome.

a All sera were applied in a 1:20 dilution and rated from negative (-), intermediate (+/-) to positive (+ until +++).

b A non-patient contact negative-control serum.

IgG depleted.

Titres (selective) were determined by serial dilutions in a range of 1:20 to 1:640.

the geographical and cultural context of our patient. Recent experimental studies have found that NCoV can infect and replicate in cells of various species including humans, swine, monkeys and bats, suggesting a more promiscuous host specificity compared to other human coronaviruses such as SARS CoV [17]. Susceptibility of goat cells was not tested, but it cannot be excluded that NCoV might infect this species as well.

Especially hospitals with ICU, specialist lung hospitals and similar facilities should consider NCoV in patients with severe respiratory disease of unknown aetiology. These patients should be tested for the novel virus as well as pathogens causing illnesses that need to be considered for differential diagnosis in severe lung disease. Full personal protective equipment such as recommended for handling patients with SARS, including N95 masks independent of the procedure performed, should be used by HCW in such cases, and responsible public health agencies should be informed timely. In general, it is prudent that HCW in contact with any patient with a severe respiratory illness of unknown origin apply droplet precautions. Should patients with suspected $\mathrm{NCoV}$ infections be transferred for special treatment it is important to fully inform the receiving hospital. Public health management recommendations should be further informed through future research that include the route, amount and duration of virus shedding. In addition, more information is needed on the ability of the virus to transmit from person to person.

Our investigation has some important limitations. We have not obtained a questionnaire and blood from all contacts of this patient. Nevertheless, response rate was high and information on contacts with the highest risk for infection was complete. Available information on the interval between exposure and venipuncture could only be approximated because contacts were exposed over more than one day. In our study we used the day of first contact because the patient was likely most infectious at this point in time. Theoretically, seroconversion may have occurred in some after contacts had provided blood. However, the need to rapidly evaluate the situation urged us to commence the contact investigation immediately. A further limitation is that the patient's negative result of virus isolation could have been due to the long storage time of the 
sample - in contrast to our favoured hypothesis of low RNA concentration.

In spite of this, we believe that it is fair to conclude the patient's infectiousness on illness day 20 was absent or very low. Our contact investigation has found no evidence of infection among hospital or out-of-hospital contacts. Our two-staged approach to serological screening where a first-line testing is done by full-virus IFA and supplemented by confirmatory recombinant IFA and SNT should provide a template for similar investigations in the future. Finally, if patients suspected to be infected with $\mathrm{NCoV}$ are to be transferred for specialised treatment, receiving hospitals need to be informed so that appropriate infection control measures can be implemented.

\section{Acknowledgements}

We thank Kerstin Prahm and Aryna Zanuzdana for support with data entry and validation, Sami Marzougi and Ghassan Moussa for their kind interpreting assistance, Annette Jurke for being the contact point at the state health department, Mareen Adam, Susi Hafemann, Ingrid Zadow and Julia Hinzmann for excellent technical assistance regarding PCR analyses and Petra Kreher and Anette Teichmann for serology test development and testing of sera. We thank Ron Fouchier of Erasmus Medical Centre for providing the virus isolate.

Work at the University of Bonn was supported by the European Union $\mathrm{FP}_{7}$ project EMPERIE (contract number 223498), ANTIGONE (contract number 278976), the German Research Foundation (DFG grant DR 772/3-1, KA1241/18-1), as well as the German Ministry of Education and Research (BMBF SARS II).

\section{Conflict of interest}

None declared.

\section{Authors' contributions}

UB: assisted in designing the study, analysed data, wrote manuscript. MAM: performed laboratory testing, read and revised manuscript. AN: performed laboratory testing, read and revised manuscript. AS: collected data, read and revised manuscript. NW: collected data, read and revised manuscript. TBB: collected data, read and revised manuscript. FB: collected data, read and revised manuscript. CD: assisted in designing the study, performed laboratory testing, read and revised manuscript. BS: performed laboratory testing, read and revised manuscript. TW: performed laboratory testing, read and revised manuscript. DM: performed laboratory testing, read and revised manuscript. BM: performed laboratory testing, read and revised manuscript. SB: assisted in designing the study, read and revised the manuscript. GK: assisted in designing the study, read and revised the manuscript. LS: assisted in designing the study, read and revised the manuscript. WH: assisted in designing the study, co-ordinated the study, read and revised the manuscript.
References

1. Zaki AM, van Boheemen S, Bestebroer TM, Osterhaus AD, Fouchier RA. Isolation of a novel coronavirus from a man with pneumonia in Saudi Arabia. N Engl J Med. 2012;367(19):181420. http://dx.doi.org/10.1056/NEJMoa1211721. PMid:23075143.

2. Centers for Disease Control and Prevention. Severe respiratory illness associated with a novel coronavirus--Saudi Arabia and Qatar, 2012. MMWR Morbidity and mortality weekly report 2012;61:820. PMid:23051613.

3. Bermingham A, Chand M, Brown C, Aarons E, Tong C, Langrish $\mathrm{C}$ et al. Severe respiratory illness caused by a novel coronavirus, in a patient transferred to the United Kingdom from the Middle East, September 2012. Euro Surveill. 2012; 17(40): pii=20290. Available from: http://www. eurosurveillance.org/ViewArticle.aspx?Articleld=20290

4. World Health Organization (WHO). Novel coronavirus infection update. Geneva: (WHO); 2013. Accessed on February 18, 2012. Available from: http://www.who.int/csr/don/2013_02_16/en/ index.html

5. European Center for Disease Prevention and Control (ECDC). Rapid risk assessment: Update: Severe respiratory disease associated with a novel coronavirus. Update 19 February 2013. Stockholm:ECDC;2013. Accessed on: February 19, 2012. Available from: http://www.ecdc.europa.eu/en/publications/ Publications/novel-coronavirus-rapid-risk-assessment-update. pdf

6. World Health Organization (WHO). Infection prevention and control of epidemic- and pandemic-prone acute respiratory diseases in health care - WHO interim guidelines. Geneva: (WHO); 2007. Accessed on: December 17, 2012. Available from: http://www.who.int/csr/resources/publications/WHO_CDS EPR_2007_6c.pdf

7. Pebody RG, Chand MA, Thomas HL, Green HK, Boddington NL, Carvalho C, et al. The United Kingdom public health response to an imported laboratory confirmed case of a novel coronavirus in September 2012. Euro Surveill. 2012;17(40):pii=20292. Available from: http://www. eurosurveillance.org/ViewArticle. aspx?Articleld $=20292$

8. World Health Organization (WHO). International health regulations (2005). Second edition. Geneva: WHO; 2008. Available from: http://whqlibdoc.who.int/ publications/2008/9789241580410_eng.pdf

9. Corman V, Eckerle I, Bleicker T, Zaki A, Landt O, EschbachBludau M, et al. Detection of a novel human coronavirus by real-time reverse-transcription polymerase chain reaction. Euro Surveill, 2012;17(39): $\mathrm{pii}=20285$. Available from: http:// www.eurosurveillance.org/ViewArticle.aspx?Articleld=20285

10. Corman VM, Müller MA, Costabel U, Timm J, Binger T, Meyer B, et al. Assays for laboratory confirmation of novel human coronavirus (hCoV-EMC) infections. Euro Surveill. 2012;17(49):pii=20334. Available from: http://www. eurosurveillance.org/ViewArticle. aspx?Articleld $=20334$

11. Herzog P, Drosten C, Müller MA. Plaque assay for human coronavirus NL63 using human colon carcinoma cells. Virol J. 2008;5:138. http://dx.doi.org/10.1186/1743-422X-5-138. PMid:19014487. PMCid:2603006.

12. Federal Ministry of Justice. Gesetz zur Verhütung und Bekämpfung von Infektionskrankheiten beim Menschen (Infektionsschutzgesetz - IfSG). [Regulation on preventing and control of infectious diseases in humans (Act on protection against infection)]. 20 Jul 2000. German. Available from: http://bundesrecht.juris.de/bundesrecht/ifsg/gesamt.pdf

13. van Boheemen S, de Graaf M, Lauber C, Bestebroer TM, Raj VS, Zaki AM, et al. Genomic characterization of a newly discovered coronavirus associated with acute respiratory distress syndrome in humans. MBio. 2012;3. pii: e00473-12. http:// dx.doi.org/10.1128/mBio.00473-12

14. Annan A, Baldwin HJ, Corman VM, Klose SM, Owusu $M$, Nkrumah $\mathrm{EE}$, et al. Human betacoronavirus $2 \mathrm{C}$ EMC/2012 $\neg$ related viruses in bats, Ghana and Europe. Emerg Infect Dis 2013 Mar (epub ahead of print). http://dx.doi. org/10.3201/eid1903.121503

15. Albarrak AM, Stephens GM, Hewson R, Memish ZA. Recovery from severe novel coronavirus infection. Saudi medical journal 2012;33:1265-9. PMid:23232672.

16. Saif LJ. Bovine respiratory coronavirus. Vet Clin North Am Food Anim Pract. 2010;26:349-64. http://dx.doi.org/10.1016/j. cvfa.2010.04.005

17. Muller MA, Raj VS, Muth D, Meyer B, Kallies S, Smits SL, et al. Human Coronavirus EMC Does Not Require the SARSCoronavirus Receptor and Maintains Broad Replicative Capability in Mammalian Cell Lines. mBio 3:eo0515-12. http:// dx.doi.org/10.1128/mBio.00515-12 